\title{
BMJ Open The impact of exacerbation frequency on mortality following acute exacerbations of COPD: a registry-based cohort study
}

Sigrun Alba Johannesdottir Schmidt, ${ }^{1}$ Martin Berg Johansen, ${ }^{1}$ Morten Olsen, ${ }^{1}$ Xiao Xu, ${ }^{2}$ Joseph M Parker, ${ }^{3}$ Nestor A Molfino, ${ }^{4}$ Timothy L Lash, ${ }^{1,5}$ Henrik Toft Sørensen, ${ }^{1}$ Christian Fynbo Christiansen ${ }^{1}$

To cite: Schmidt SAJ, Johansen MB, Olsen M, et al. The impact of exacerbation frequency on mortality following acute exacerbations of COPD: a registry-based cohort study. BMJ Open 2014;4:e006720.

doi:10.1136/bmjopen-2014006720

- Prepublication history and additional material is available. To view please visit the journal (http://dx.doi.org/ 10.1136/bmjopen-2014006720).

Received 23 September 2014 Revised 1 December 2014 Accepted 2 December 2014

CrossMark

For numbered affiliations see end of article.

Correspondence to Dr Sigrun Alba Johannesdottir Schmidt; saj@clin.au.dk

\section{ABSTRACT}

Objective: To examine the association between exacerbation frequency and mortality following an acute exacerbation of chronic obstructive pulmonary disease (AECOPD).

Design: Cohort study using medical databases. Setting: Northern Denmark.

Participants: On 1 January 2005, we identified all patients with prevalent hospital-diagnosed chronic obstructive pulmonary disease (COPD) who had at least one AECOPD during 1 January 2005 to 31 December 2009. We followed patients from the first AECOPD during this period until death, emigration or 31 December 2009, whichever came first. We flagged all AECOPD events during follow-up and characterised each by the exacerbation frequency $(0,1,2$ or $3+)$ in the prior 12-month period.

Main outcomes and measures: Using Cox regression, we computed 0-30-day and 31-365-day age-adjusted, sex-adjusted, and comorbidity-adjusted mortality rate ratios (MRRs) with $95 \% \mathrm{Cls}$ entering exacerbation frequency as a time-varying exposure.

Results: We identified 16647 eligible patients with prevalent COPD, of whom 6664 (40\%) developed an AECOPD and were thus included in the study cohort. The 0-30-day MRRs were 0.97 (95\% $\mathrm{Cl} 0.80$ to 1.18), $0.90(95 \% \mathrm{Cl} 0.70$ to 1.15$)$ and $1.03(95 \% \mathrm{Cl} 0.81$ to 1.32) among patients with AECOPD with 1,2 and $3+$ AECOPDs versus no AECOPD within the past 12 months, respectively. The corresponding MRRs were 1.47 (95\% Cl 1.30 to 1.66$), 1.89$ (95\% Cl 1.59 to 2.25$)$ and $1.59(95 \% \mathrm{Cl} 1.23$ to 2.05$)$ for days 31-365.

Conclusion: Among patients with AECOPD, one or more exacerbations in the previous year were not associated with 30-day mortality but were associated with an increased 31-365-day mortality.

\section{INTRODUCTION}

Chronic obstructive pulmonary disease (COPD) is characterised by a progressive

\section{Strengths and limitations of this study}

- The universal healthcare system and complete follow-up of all residents reduces the risk of selection bias.

- The study examined the association according to chronic obstructive pulmonary disease (COPD) treatment at study start and found no substantial variation across treatment groups.

- The broad definitions included patients with hospital-diagnosed COPD treated for acute exacerbations of COPD (AECOPD) outside the hospital setting. However, COPD patients treated in general practice exclusively were not included. Also, the use of a prevalent cohort may have resulted in a mix of patients at different stages in their clinical course of COPD.

- The study lacked information on clinical variables, which would have been useful in classifying AECOPD and elucidating the association.

- Excess non-COPD mortality may explain the association observed after 30 days of follow-up.

decline in pulmonary function due to airway inflammation in response to noxious particles and gases. ${ }^{12}$ In Denmark, the standardised incidence rate of hospitalisation for COPD was 231/100 000 person-years in 2006. ${ }^{3}$ The $0-180$-day and 181-day to 5-year standardised mortality rates in patients with COPD were 389/1000 person-years and 164/ 1000 person-years, respectively, ${ }^{3}$ making it one of the leading causes of death among the elderly. ${ }^{4}$

COPD is frequently complicated by acute exacerbations (AECOPD), defined as "an acute event characterised by a worsening of the patient's respiratory symptoms that is beyond normal day-to-day variations and leads to change in medication." 5 The annual number of exacerbations in patients with COPD is estimated at between 0.82 and 
2.01. ${ }^{6} 7$ Exacerbation frequency ${ }^{6}$ and mortality ${ }^{8}$ rates rise with increasing COPD severity. However, frequent AECOPDs may themselves result in decreased lung function and could thereby increase disease severity and AECOPD mortality. ${ }^{1289}$ Several epidemiological studies support this by demonstrating an impact of exacerbation history on mortality in patients admitted with AECOPD. Comparison of previous studies is, however, hampered by differences in the definitions of AECOPD frequency, in the length of follow-up and in the patient populations included. $^{10-19}$ Furthermore, none of the studies included AECOPDs treated outside the hospital. Finally, only one of the studies examined if the association depended on preadmission therapy. ${ }^{11}$ Unfortunately, authors provided only an insignificant interaction term for the analysis, ${ }^{11}$ which limits the interpretation to statistical significance only.

We conducted a cohort study to examine how the exacerbation frequency impacts 1-year mortality following an AECOPD. Specifically, we addressed the limitations of previous studies by including exacerbations treated in the hospital, outpatient clinics and in general practice, and by using Danish registries with detailed data on comorbidity, COPD treatment and with complete follow-up.

\section{METHODS}

\section{Setting and data sources}

We conducted this cohort study in northern Denmark, which has a population of approximately 1.8 million (30\% of the Danish population). In Denmark, a taxsupported healthcare plan guarantees universal medical care for all residents and partial reimbursement for prescribed medications. ${ }^{20}$ Virtually all health services are recorded in various medical registries, of which the following formed the basis for this study. The Danish National Registry of Patients (DNRP) has maintained records on all inpatient admissions to non-psychiatric hospitals since 1977, and on all outpatient specialist clinic and emergency room visits since $1995 .^{21}$ Each admission is described by one primary diagnosis and one or more secondary diagnoses classified according to the eighth revision of the International Classification of Diseases (ICD-8) through 1993 and the ICD-10 revision thereafter. $^{21}$

Aarhus University Prescription Database records the patient's personal identifier, the dispensing date, and the type and quantity of drug prescribed (according to the Anatomical Therapeutic Chemical (ATC) Classification System) each time a prescription is redeemed at the pharmacy. ${ }^{22}$

Since 1968, the Danish Civil Registration System has recorded all Danish residents' administrative information and changes in vital status, such as date of death and emigration, with daily updates. ${ }^{20}$ The registry assigns a unique personal identifier to all persons born in or immigrating to Denmark, which enables follow-up of patients and linkage of the various medical registries. $^{20}$

All codes used for defining study variables in the current study can be found in eTable 1 .

\section{Study population}

The population eligible for the study included all patients with prevalent COPD on 1 January 2005, who had a COPD diagnosis recorded in the DNRP between 1 January 1995 and 31 December 2004. We considered all primary inpatient and outpatient diagnosis related to COPD as well as all primary diagnoses of respiratory failure with a secondary COPD-related diagnosis, as described previously ${ }^{23}$ and defined in the online supplementary file. Patients aged younger than 40 years were excluded, given the low COPD prevalence in this patient group $^{24}$ and the potential for misclassifying asthma as COPD.

Among all eligible patients with COPD, we then identified the study cohort as patients with COPD who developed at least one AECOPD between 1 January 2005 and 31 December 2009. We used the DNRP and the Aarhus University Prescription Database to identify acute exacerbations as (a) a redemption of a systemic glucocorticoid prescription and an antibiotic prescription on the same day (to account for patients treated outside hospital) or (b) a primary hospital discharge diagnosis of AECOPD or (c) a primary hospital discharge diagnosis of respiratory failure or acute respiratory infection with a secondary discharge diagnosis of AECOPD. We did not include emergency room diagnoses of COPD or AECOPD in this study, as COPD is rarely treated in this setting in Denmark (only $1 \%$ of AECOPD cases were treated exclusively in the emergency department). Standard practice at Danish hospitals is to admit patients with AECOPD directly to the acute admission unit. Also, patients with COPD who are transferred from the emergency room to a specialised ward are coded as inpatient admissions and are therefore included in the study.

Using the Civil Registration System, we followed patients from the date of first exacerbation recorded between 1 January 2005 and 31 December 2009 and continued until death, emigration, or 31 December 2009, whichever came first. To examine the effect of AECOPD frequency on mortality, we classified each AECOPD during follow-up according to whether it was preceded by $0,1,2$ or $3+$ AECOPDs in the prior 12 months. We then entered this value as a time-varying exposure in the analysis. Therefore, each time a patient had an AECOPD during follow-up, we assessed the number of AECOPDs in the 12 months before the event and assigned the patient to the corresponding exposure group (0, 1, 2 or 3+ AECOPDs). One patient could thus have multiple AECOPDs during follow-up and contribute person-time in several exposure groups depending on the rate of AECOPD. We adjudicated AECOPD events using a 30-day threshold following the prescription redemption or hospitalisation, that is, an AECOPD 
event within 30 days of a previous AECOPD was not regarded as a new AECOPD.

\section{Covariates}

We used the DNRP to retrieve the hospital history for all study participants during the 5 years preceding the start of the study on 1 January 2005 . We then ascertained the presence of the following diseases that are frequent among patients with COPD and may affect mortality: myocardial infarction, congestive heart failure, peripheral vascular disease, cerebrovascular disease, peptic ulcer disease, liver disease, diabetes, moderate to severe renal disease, any malignancy except lung cancer, alcoholism-related diseases, atrial fibrillation/flutter, medically diagnosed obesity, hypertension, osteoporosis, lung cancer, asthma, obstructive sleep apnoea, venous thromboembolism, rheumatoid arthritis and depression.

From the Prescription Database, we retrieved information on COPD treatment within 12 months before study start. Following the latest Global Initiative for Chronic Obstructive Lung Disease (GOLD) guidelines, ${ }^{5}$ we then grouped patients into the following five mutually exclusive groups of escalating treatment: (1) non-treated/ unclassified, (2) short-acting bronchodilators, (3) monotherapy with a long-acting bronchodilator ( $\beta_{2}$-agonists or long-acting muscarinic antagonists), (4) double therapy with any possible combination of long-acting $\beta_{2}$-agonists, inhaled corticosteroids and/or long-acting muscarinic antagonists and (5) triple therapy with long-acting $\beta_{2}$-agonists, inhaled corticosteroids and/or long-acting muscarinic antagonists, as defined in eTable 1 . We also retrieved information on pharmacological treatment with systemic corticosteroids or theophylline within 12 months before study start, with antibiotics and/or antivirals within 3 months before study start. Finally, we used the DNRP to identify hospital codes for oxygen treatment within the 12 months before study start and on lung volume reduction surgery between 1996 and study start.

\section{Statistical analysis}

We characterised the eligible population of patients with COPD on 1 January 2005 by age, sex, comorbidities recorded in the 5 years before study start, as well as COPD treatment group, pharmacological treatment with systemic steroids, theophylline or oxygen therapy within the prior 12 months, and treatment with antibiotics and/or antivirals within the prior 3 months.

In the mortality analyses, we entered AECOPD frequency as a time-varying exposure and computed the number of deaths, person-time and mortality rates in each exposure group. We then used Cox regression analysis to compute crude hazard ratios as a measure of mortality rate ratios (MRRs) and associated 95\% CIs for patients with AECOPD with 1, 2, or 3+ AECOPDs in the 12 months preceding an AECOPD, compared with patients with no exacerbations in the preceding 12-month period. We then computed the MRRs adjusted for sex, age (as a continuous variable) and comorbidities. In a subanalysis, we examined the effect of frequent severe exacerbations on mortality by including only severe AECOPDs, which were defined as inpatient admissions for AECOPD ((b) or (c) listed above). The present AECOPD and any exacerbations in the 12 months before, both had to be defined as severe. Finally, we stratified the results from the primary analysis and the results for severe AECOPD only by age group at study start, sex, and presence/absence of oxygen therapy, lung volume reduction surgery, COPD treatment group and cardiovascular disease (myocardial infarction, congestive heart failure, peripheral vascular disease and cerebrovascular disease).

Because AECOPD is an acute event, we expect the mortality rate to be greatest in the initial phase following the event. We therefore separated the effect of AECOPD on mortality in the first 30 days versus day 31 to day 365 after the event in all mortality rate calculations. We assessed the assumption of proportional hazards graphically using scaled Schoenfeld residuals and found it valid.

All analyses were performed using SAS V.9.2 (SAS Institute Inc, Cary, North Carolina, USA). The study was approved by the Danish Data Protection Agency (journal number 2013-41-1924).

\section{RESULTS}

\section{Descriptive data}

We identified 16647 patients with COPD eligible for the study on 1 January 2005. Median age among eligible patients was 70 years and $53 \%$ were female (table 1). Comorbidities were frequent, especially cardiovascular disease, diabetes, osteoporosis and asthma. A high proportion of patients had redeemed prescriptions for antibiotics within the previous 3 months (31\%) and for systemic steroids within the previous 12 months $(30 \%)$. The majority of patients received double therapy. Among the 16647 eligible patients with COPD, 6664 (40\%) had at least one AECOPD during the subsequent 5 years and thus constituted the study population for our study.

\section{Mortality following AECOPD}

The 30-day all-cause mortality rate following an AECOPD was 552, 485, 441 and 477/1000 person-years for $0,1,2$ and 3+ AECOPDs in the prior 12 months, respectively (table 2). Compared with patients with no AECOPD in the prior 12 months, the mortality rate did not depend on the frequency of events. Thus, the MRR was $0.97(95 \%$ CI 0.80 to 1.18$)$ for 1 AECOPD, 0.90 (95\% CI 0.70 to 1.15 ) for 2 AECOPDs and 1.03 (95\% CI 0.81 to 1.32 ) for $3+$ AECOPDs in the prior 12 months (table 2).

The absolute mortality rate decreased substantially after day 30 and was higher among patients with one or more AECOPDs in the 12 months preceding their 
Table 1 Characteristics of eligible patients with prevalent COPD for the study on 1 January 2005

\begin{tabular}{|c|c|c|}
\hline Characteristic & $\mathbf{n}$ & Per cen \\
\hline Total & 16647 & 100 \\
\hline \multicolumn{3}{|l|}{ Age at study start (years)* } \\
\hline $40-50$ & 1198 & 7.2 \\
\hline $50-60$ & 2764 & 17 \\
\hline $60-70$ & 4522 & 27 \\
\hline $70-80$ & 5422 & 33 \\
\hline 80-90 & 2492 & 15 \\
\hline $90+$ & 249 & 1.5 \\
\hline \multicolumn{3}{|l|}{ Sex } \\
\hline Female & 8770 & 53 \\
\hline Male & 7877 & 47 \\
\hline \multicolumn{3}{|c|}{ Comorbidities (within previous 5 years) } \\
\hline Myocardial infarction & 795 & 4.8 \\
\hline Congestive heart failure & 1785 & 11 \\
\hline Peripheral vascular disease & 922 & 5.5 \\
\hline Cerebrovascular disease & 1178 & 7.1 \\
\hline Peptic ulcer disease & 627 & 3.8 \\
\hline Liver disease & 176 & 1.1 \\
\hline Diabetes & 1134 & 6.8 \\
\hline Moderate to severe renal disease & 287 & 1.7 \\
\hline Any malignancy except lung cancer & 950 & 5.7 \\
\hline Alcoholism-related diseases & 162 & 1.0 \\
\hline Atrial fibrillation/flutter & 1400 & 8.4 \\
\hline Medically diagnosed obesity & 575 & 3.5 \\
\hline Hypertension & 2066 & 12 \\
\hline Osteoporosis & 1021 & 6.1 \\
\hline Lung cancer & 186 & 1.1 \\
\hline Asthma & 2006 & 12 \\
\hline Obstructive sleep apnoea & 337 & 2.0 \\
\hline Rheumatoid arthritis & 151 & 0.9 \\
\hline Depression & 340 & 2.0 \\
\hline \multicolumn{3}{|l|}{ Treatments within previous 12 months } \\
\hline Systemic steroids & 4993 & 30 \\
\hline Theophylline & 1164 & 7.0 \\
\hline Oxygen therapy & 258 & 1.5 \\
\hline \multicolumn{3}{|l|}{ COPD treatment } \\
\hline Non-treated/unclassified & 4880 & 29 \\
\hline Short-acting bronchodilators & 2958 & 18 \\
\hline Long-acting bronchodilator & 2041 & 12 \\
\hline Double therapy & 4226 & 25 \\
\hline Triple therapy & 2542 & 15 \\
\hline \multicolumn{3}{|l|}{ Infection within previous 3 months } \\
\hline Prescription for antibiotics & 5103 & 31 \\
\hline Prescription for antivirals & 55 & 0.3 \\
\hline $\begin{array}{l}\text { Prescription for antibiotics as well as } \\
\text { antivirals }\end{array}$ & 26 & 0.2 \\
\hline
\end{tabular}

See the text and online supplementary appendix for definition of GOLD treatment groups.

Previous lung volume reduction surgery is not shown in the table because it was rare (close to $0 \%$ ).

*Overall, the median age was 70 years (lower quartile 61 years; upper quartile 77 years).

COPD, chronic obstructive pulmonary disease; GOLD, Global Initiative for Chronic Obstructive Lung Disease.

AECOPD (table 2). Thus, the MRRs were 1.47 (95\% CI 1.30 to 1.66$), 1.89$ (95\% CI 1.59 to 2.25$)$ and $1.59(95 \%$ CI 1.23 to 2.05) among patients with AECOPD who had experienced 1, 2 and 3+ AECOPDs compared with no
AECOPD in the 12 months before the AECOPD event, respectively.

Severe AECOPDs (requiring hospitalisation) were associated with higher absolute mortality rates than AECOPDs overall, in particular for the 0-30-day period (table 3). However, the relative impact of AECOPD frequency was similar to the overall results.

The stratified main analyses (eTable 2) showed that for the 31-365-day period, the MRRs were highest among those aged 50-59 years $(\mathrm{MRR}=2.13,95 \%$ CI 1.34 to 3.41 for $1 \mathrm{AECOPD}$; MRR=2.14, 95\% CI 1.07 to 4.26 for 2 AECOPDs; and MRR=3.43, 95\% CI 1.64 to 7.15 for $3+$ AECOPDs) and those with oxygen therapy within 12 months before study start $(\mathrm{MRR}=3.61,95 \%$ CI 1.80 to 7.27 for 1 AECOPD; MRR=4.87, 95\% CI 1.79 to 13.2 for 2 AECOPDs; and MRR=4.22, 95\% CI 0.82 to 21.7 for $3+$ AECOPDs). There was no substantial variation by COPD treatment. The stratified analysis considering severe AECOPDs only (eTable 3) were similar to the stratification including all AECOPDs regardless of severity. However, the stratified analyses should be interpreted with the wide CIs in mind.

\section{DISCUSSION}

In this large Danish cohort study, we found that the 31365-day all-cause mortality rate following an AECOPD was higher among patients with at least one AECOPD in the preceding 12 months than among patients who did not have any recent AECOPD. All cause mortality did not vary substantially according to the actual number of AECOPDs within the preceding year and was not observed in excess within the first 30 days after the AECOPD after controlling for age, sex and comorbidities.

\section{Strengths and limitations}

The universal healthcare system and complete follow-up of all residents by the Danish Civil Registration System reduces the possibility of selection bias in our study. We aimed to reduce information bias by using broad definitions of COPD and AECOPD rather than more restrictive primary discharge diagnoses. A review of medical records has shown a positive predictive value (PPV) of 93\% for primary COPD diagnoses in the DNRP and a PPV of $92 \%$ when also including primary diagnoses of respiratory failure or pneumonia with secondary COPD diagnosis. $^{25}$ However, by using prescriptions for a systemic glucocorticoid and an antibiotic redeemed on the same day to define AECOPDs outside the hospital setting, we may have misclassified some patients who did not have AECOPD as patients with AECOPD if the prescriptions were written as rescue packs for potential future events. Such misclassification would not affect the analysis restricted to only severe AECOPDs. Also, our choice of an arbitrary duration of 30 days may have resulted in misclassification of exposure status due to underreporting or misclassification of the number of 
Table 2 Mortality following an AECOPD according to the number of exacerbations in the previous year

\begin{tabular}{|c|c|c|c|c|}
\hline $\begin{array}{l}\text { Frequency of AECOPD in the } \\
12 \text { months prior to an AECOPD }\end{array}$ & $\begin{array}{l}\text { Number of } \\
\text { deaths }\end{array}$ & Person-years & $\begin{array}{l}\text { Mortality rate and } 95 \% \mathrm{Cl} \\
\text { (per } 1000 \text { person-years) }\end{array}$ & $\mathrm{HR}$ and $95 \% \mathrm{Cl}^{*}$ \\
\hline \multicolumn{5}{|l|}{ 0-30 days } \\
\hline 0 & 303 & 581 & 522 (466 to 584$)$ & (ref.) \\
\hline 1 & 164 & 338 & 485 (416 to 565$)$ & 0.97 (0.80 to 1.18$)$ \\
\hline 2 & 79 & 179 & 441 (354 to 550 ) & 0.90 (0.70 to 1.15$)$ \\
\hline $3+$ & 86 & 180 & 477 (386 to 589 ) & 1.03 (0.81 to 1.32 ) \\
\hline \multicolumn{5}{|l|}{31 and up to 365 days } \\
\hline 0 & 933 & 5830 & $160(150$ to 171$)$ & (ref.) \\
\hline 1 & 359 & 1573 & 228 (206 to 253 ) & 1.47 (1.30 to 1.66$)$ \\
\hline 2 & 146 & 5205 & 281 (239 to 331 ) & 1.89 (1.59 to 2.25$)$ \\
\hline $3+$ & 63 & 266 & 237 (185 to 303$)$ & 1.59 (1.23 to 2.05$)$ \\
\hline
\end{tabular}

Northern Denmark, 2005-2009.

*Adjusted for age (as a continuous variable), sex and comorbidities.

AECOPD, acute exacerbation of chronic obstructive pulmonary disease.

AECOPDs. Previous data, however, show that the majority of patients recover within 30 days after AECOPD onset. $^{26}$

Because we relied solely on registry data, we lacked information on lifestyle factors and clinical variables, such as measurements of peak expiratory flow and arterial blood gases. However, some of the clinical factors may be on the causal pathway linking AECOPD fre-

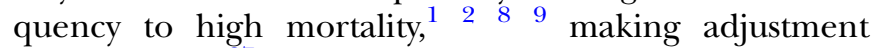
inappropriate. ${ }^{27}$ Nevertheless, such information would have been useful in classifying AECOPD. Instead, we examined if the association depended on COPD therapy, which may be linked to underlying severity, and found no evidence thereof. A total of $29 \%$ in the eligible cohort were non-treated/unclassified, which may represent patients with poor adherence or possibly patients with mild COPD.

Finally, the generalisability of our results to the entire range of AECOPD cases may be affected by the fact that some patients with COPD may be diagnosed in general practice, excluding them from registration in the DNRP.

\section{Comparison with other studies}

In a Canadian inception cohort of 73106 patients with COPD, Suissa $e t a l^{17}$ showed that the AECOPD mortality rate increased with each exacerbation, as compared with the mortality rate following the first AECOPD. The adjusted MRR was 1.9 (95\% CI 1.8 to 1.9 ) for the second AECOPD increasing to 5.2 (95\% CI 4.9 to 5.5) after the 10th or later events. Mortality peaked within the first week after admission. Several other studies have also found an association between a history of AECOPD and mortality. ${ }^{10-16} 1819$ However, definitions of exposure have varied greatly, including a history of hospitalisation for AECOPD within 6 months, ${ }^{16} 1$ year, ${ }^{10} 11141819$ and up to 7 years ${ }^{12}$ before current AECOPD hospitalisation, within 2 years before inclusion period, ${ }^{15}$ or admission with respiratory failure within 2 years before current admission. ${ }^{13}$ Similarly, various definitions of AECOPD mortality were applied, including mortality in-hospital, ${ }^{12} 13 \quad 16$ at 30 days $^{13}$ and at longer term (median 3.1 years) ${ }^{14}$ following admission, and at 3 months, ${ }^{10} 6$ months, ${ }^{11} 1$ year, ${ }^{11} 2$ years ${ }^{11}{ }^{19}$ and at longer

Table 3 Mortality following an AECOPD according to the number of exacerbations in the previous year

\begin{tabular}{|c|c|c|c|c|}
\hline $\begin{array}{l}\text { Frequency of severe AECOPD in } \\
\text { the } 12 \text { months prior to a severe } \\
\text { AECOPD }\end{array}$ & $\begin{array}{l}\text { Number of } \\
\text { deaths }\end{array}$ & Person-years & $\begin{array}{l}\text { Mortality rate and } 95 \% \mathrm{Cl} \text { (per } \\
1000 \text { person-years) }\end{array}$ & $\mathrm{HR}$ and $95 \% \mathrm{Cl}^{*}$ \\
\hline \multicolumn{5}{|l|}{$0-30$ days } \\
\hline 0 & 283 & 301 & 939 (836 to 1055$)$ & (ref.) \\
\hline 1 & 109 & 114 & 954 (790 to 1151$)$ & $1.07(0.85$ to 1.33$)$ \\
\hline 2 & 52 & 46 & 1123 (855 to 1473 ) & $1.29(0.96$ to 1.75$)$ \\
\hline $3+$ & 32 & 36 & 893 (631 to 1262$)$ & $1.09(0.75$ to 1.59$)$ \\
\hline \multicolumn{5}{|l|}{31 and up to 365 days } \\
\hline 0 & 648 & 2974 & 218 (201 to 235 ) & (ref.) \\
\hline 1 & 196 & 523 & 375 (326 to 431$)$ & 1.75 (1.49 to 2.06$)$ \\
\hline 2 & 51 & 146 & 349 (265 to 459$)$ & $1.67(1.26$ to 2.23$)$ \\
\hline $3+$ & 22 & 59 & 371 (245 to 564$)$ & 1.77 (1.15 to 2.72$)$ \\
\hline
\end{tabular}

Northern Denmark, 2005-2009. Only severe (hospitalised) AECOPDs included.

*Adjusted for age (as a continuous variable), sex and comorbidities.

AECOPD, acute exacerbation of chronic obstructive pulmonary disease. 
term mortality (3 or more years) ${ }^{15}{ }^{18}$ following discharge. Besides these differences in assessment of prior AECOPD hospitalisations and in follow-up periods, populations included also varied substantially (eg, inclusion of primarily men, ${ }^{11} 1518$ emergency room patients only $^{1416}$ and discharged patients only ${ }^{10} 151819$ ).

Surprisingly, we found no relative effect of prior AECOPD on mortality within 0-30 days following AECOPD. Also, the excess 31-365-day mortality associated with one AECOPD in the previous year was less pronounced than in the study by Suissa $e t a l^{17}$ and it did not increase further with increasing number of AECOPDs. There may be several potential explanations for this discrepancy. First, our study population included patients with prevalent COPD at study start, which may have resulted in a mix of patients at different stages in their clinical course of COPD. Hence, by mixing patients who were experiencing their first AECOPD ever with patients who had previously experienced one or more AECOPDs, we may have obscured some of the effect of AECOPD frequency on mortality. Second, unmeasured severity of the AECOPD may have affected our results. We have previously shown that patients with no AECOPD in the year before an AECOPD are younger and have less comorbidity. ${ }^{23}$ Even though these patients may have had more newly diagnosed, and thus less severe, COPD, it is possible that some of these patients have more severe AECOPDs because they postpone seeking medical attention due to unfamiliarity with the symptoms thereof. However, an older patient with higher comorbidity and a recent history of AECOPD may be more aware of the threatening situation and act more quickly, resulting in a lower mortality than expected in the acute phase. The situation may then reverse after day 30 , when the relative impact of frequent exacerbations on severity of COPD, complication rate and relapse rate becomes clearer, as well as death from causes other than COPD. Third, the study population examined by Suissa $e t a l^{17}$ included a higher proportion of men who were on average older than our study population. Thus, excess cardiovascular mortality in their population may partly explain the higher estimates observed in their study. Finally, because the absolute 30-day mortality rate was very high, but decreased substantially thereafter, it is possible that the relative effect of AECOPD history appeared less pronounced in the first period merely because of differences in the baseline rate. Such differences may also explain the more pronounced associations observed for the younger patients in our subanalysis.

\section{CONCLUSIONS}

In this cohort of patients with AECOPD, we found that a history of at least one AECOPD in the 12 months before exacerbation may serve as an indicator of a higher mortality rate during 31 to 365 days but not during the first 30 days following the AECOPD. The lack of an effect on 0-30-day mortality may be explained by study factors such as the use of a prevalent cohort of patients with COPD or a higher baseline rate than in the 31-365-day period.

\section{Author affiliations}

${ }^{1}$ Department of Clinical Epidemiology, Aarhus University Hospital, Aarhus N, Denmark

${ }^{2}$ AstraZeneca LP, Gaithersburg, Maryland, USA

${ }^{3}$ Medimmune LLC, Gaithersburg, Maryland, USA

${ }^{4}$ KaloBios Pharmaceuticals, Inc, South San Francisco, California, USA

${ }^{5}$ Department of Epidemiology, Rollins School of Public Health, Emory University, Atlanta, Georgia, USA

Contributors All authors participated in designing the study; discussion and interpretation of the results; critically revised the manuscript for intellectual content and approved the final version. MBJ collected the data and carried out analyses. SAJS organised the writing and wrote the initial draft. HTS is the guarantor.

Funding This work was supported by the Clinical Epidemiological Research Foundation and a grant from MedImmune LLC.

Competing interests XX and JMP are employed at AstraZeneca LP/ Medlmmune LLC. NAM was an employee of Medlmmune, LLC at the time of the study.

Provenance and peer review Not commissioned; externally peer reviewed.

Data sharing statement No additional data are available.

Open Access This is an Open Access article distributed in accordance with the Creative Commons Attribution Non Commercial (CC BY-NC 4.0) license, which permits others to distribute, remix, adapt, build upon this work noncommercially, and license their derivative works on different terms, provided the original work is properly cited and the use is non-commercial. See: http:// creativecommons.org/licenses/by-nc/4.0/

\section{REFERENCES}

1. Donaldson GC, Wedzicha JA. COPD exacerbations.1: Epidemiology. Thorax 2006;61:164-8.

2. Decramer M, Janssens W, Miravitlles M. Chronic obstructive pulmonary disease. Lancet 2012;379:1341-51.

3. Lash TL, Johansen MB, Christensen S, et al. Hospitalization rates and survival associated with COPD: a nationwide Danish cohort study. Lung 2010;189:27-35.

4. Juel K, Drøssing M. [Chronic obstructive pulmonary disease]. Public Health Report, Denmark, 2007.

5. From the Global Initiative for Chronic Obstructive Lung Disease: Global Strategy for the Diagnosis, Management, and Prevention of Chronic Obstructive Pulmonary Disease (updated 2013). http://www. goldcopd.org/ (accessed 28 Apr 2013).

6. Hoogendoorn M, Feenstra TL, Hoogenveen RT, et al. Association between lung function and exacerbation frequency in patients with COPD. COPD 2010;5:435-44.

7. Hurst JR, Vestbo J, Anzueto A, et al. Susceptibility to exacerbation in chronic obstructive pulmonary disease. $N$ Engl J Med 2010;363:1128-38.

8. Seemungal TAR, Hurst JR, Wedzicha JA. Exacerbation rate, health status and mortality in COPD-a review of potential interventions. COPD 2009;4:203-23.

9. Wedzicha JA, Brill SE, Allinson JP, et al. Mechanisms and impact of the frequent exacerbator phenotype in chronic obstructive pulmonary disease. BMC Med 2013;11:181.

10. Almagro P, Cabrera FJ, Diez J, et al. Comorbidities and short-term prognosis in patients hospitalized for acute exacerbation of COPD: the EPOC en Servicios de medicina interna (ESMI) study. Chest 2012;142:1126-33.

11. Almagro $P$, Calbo $E$, Ochoa de Echagüen $A$, et al. Mortality after hospitalization for COPD. Chest 2002;121:1441-8.

12. Dransfield MT, Rowe SM, Johnson JE, et al. Use of $\beta$ blockers and the risk of death in hospitalised patients with acute exacerbations of COPD. Thorax 2008;63:301-5. 
13. Faustini A, Marino C, D'Ippoliti D, et al. The impact on risk-factor analysis of different mortality outcomes in COPD patients. Eur Respir J 2008;32:629-36.

14. Kim S, Clark S, Camargo CA Jr. Mortality after an emergency department visit for exacerbation of chronic obstructive pulmonary disease. COPD 2006;3:75-81.

15. McGhan $\mathrm{R}$, Radcliff $\mathrm{T}$, Fish $\mathrm{R}$, et al. Predictors of rehospitalization and death after a severe exacerbation of COPD. Chest 2007;132:1748-55.

16. Roche N, Zureik M, Soussan D, et al. Predictors of outcomes in COPD exacerbation cases presenting to the emergency department. Eur Respir J 2008;32:953-61.

17. Suissa S, Dell'Aniello S, Ernst P. Long-term natural history of chronic obstructive pulmonary disease: severe exacerbations and mortality. Thorax 2012;67:957-63.

18. Piquet J, Chavaillon JM, David P, et al. High-risk patients following hospitalisation for an acute exacerbation of COPD. Eur Respir $J$ 2013;42:946-55.

19. Gudmundsson G, Gislason T, Lindberg E, et al. Mortality in COPD patients discharged from hospital: the role of treatment and co-morbidity. Respir Res 2006;7:109.
20. Schmidt M, Pedersen L, Sørensen HT. The Danish civil registration system as a tool in epidemiology. Eur J Epidemiol 2014;29:541-9.

21. Lynge E, Sandegaard JL, Rebolj M. The Danish national patient register. Scand J Public Health 2011;39:30-3.

22. Ehrenstein V, Antonsen S, Pedersen L. Existing data sources for clinical epidemiology: Aarhus University Prescription Database. Clin Epidemiol 2010;2:273-9.

23. Johannesdottir SA, Christiansen CF, Johansen MB, et al Hospitalization with acute exacerbation of chronic obstructive pulmonary disease and associated health resource utilization: a population-based Danish cohort study. J Med Econ 2013;16:897-906.

24. Littner MR. In the clinic. Chronic obstructive pulmonary disease. Ann Intern Med 2011;154:ITC4-1- 15; quiz ITC4-16.

25. Thomsen RW, Lange P, Hellquist $B$, et al. Validity and underrecording of diagnosis of COPD in the Danish National Patient Registry. Respir Med 2011;105:1063-8.

26. Burge S, Wedzicha JA. COPD exacerbations: definitions and classifications. Eur Respir J 2003;21:46s-53s.

27. Schisterman EF, Cole SR, Platt RW. Overadjustment bias and unnecessary adjustment in epidemiologic studies. Epidemiology 2009;20:488-95. 